\title{
Effect of Various Parameters on Bio-Synthesis of Copper Nanoparticles Using Citrus Medica Linn (Lemon) Extract and Its Antibacterial Activity
}

\author{
Sharmila Pradhan ${ }^{1,2^{*}}$, Rajeswori Shrestha ${ }^{1}$, Khuma Bhandari ${ }^{1}$ \\ ${ }^{1}$ Amrit campus, Tribhuvan University, Kathmandu, Nepal \\ ${ }^{2}$ Nepal Polymer Institute, Kirtipur, Nepal \\ "Email:amatyasharmila@yahoo.com sharmilapradhan23@gmail.com
}

(Received: 23 April, 2020, Received in revised form: 7 August, 2020, Accepted: 14 August, 2020, Available online)

\section{Highlights}

- Bio-synthesis of CuNPs using lemon extract.

- Effect of volume of extract, concentration and temperature on biosynthesis

- Antibacterial activity of CuNPs was studied via agar well diffusion method.

\begin{abstract}
This research is focused on bio-synthesis of Copper nanoparticles (CuNPs) using lemon extract to study the effect of various parameters on synthesis and to explore antibacterial activity. The biomlecules present in lemon extract act as self reducing and stabilizing agent. The synthesis of CuNPs was found to be affected by various parameters like volume of the lemon extract, concentration of the precursor and the temperature etc. Preliminary characterization of formation of nanoparticles were done by color change and UV-visible (UV-vis) spectroscopy. Elemental composition of the prepared sample was determined via Energy Dispersive X-ray (EDX) Spectroscopy. Presence of important functional groups associated with biomolecules is well characterized by Fourier Transform Infrared spectroscopy (FTIR). Scanning Electron Microscopy (SEM) revealed the formation agglomerated CuNPs of different shape and sizes and the X-ray diffraction pattern showed the formation of purely crystalline nature of CuNPs. Finally, agar well diffusion method showed that CuNPs have potential antibacterial activity against Gram-ve bacteria compared to Gram + ve bacteria.
\end{abstract}

Keywords: Bio-synthesis, CuNps, Precursor, XRD, FTIR, Agar well diffusion

\section{Introduction}

People have been much aware of the problem of environmental degradation since last decade of the $20^{\text {th }}$ century. Considering, the problem of environment, most of the research has been focused on environmentally benign method employing, green materials. Metal nanoparticle is thought to be one of the current topics of research in the field of nano-science for their wide scope of applications such as in the magnetic recording media or microelectronics, nano-medicine, optoelectronic, nanoelectronics, nanosensors, photonics, catalysis and information storage devices (Ashtaputrey et al. 2017; Prathna et al. 2016).

Among various types of metallic nanoparticles, copper is the most widely used material in the field of nanoscience due to its electrical, optical, catalytic, biomedical, antifungal, and antibacterial properties. Copper nanoparticles (CuNPs) have been extremely employed in various sectors such as water treatment, pharmacology, medical fields, targeted drug delivery, cancer treatment, gene therapy, DNA analysis, antibacterial agents, biosensors, enhancing reaction etc (Kulkarni \& Kulkarni 2013;

*Corresponding author 
Padma et al. 2018; Dinda et al. 2015). Copper-based compounds have been used in electronics since ancient times in electronic circuits due to its excellent electrical conductivity and low cost (Suramwar et al. 2016). As a consequence of possessing vital conducting properties, good compatibility, and surface-enhanced raman scattering activity, $\mathrm{Cu}$ and $\mathrm{Cu}$ based compounds will receive much demand in future nanodevices (Suramwar et al. 2016; Mittu 2016). The more important aspect is that Copper nanoparticles (CuNPs) are cheaper compared to gold, silver nanoparticles, and their properties can be controlled depending on the synthesis method and are affected by different parameters while synthesizing the materials (Karthik \& Geetha 2013; Padma et al. 2018). Hence, the synthesis and exploration of different properties of CuNPs is still of high demand.

Various methods such as vapour deposition, radiolysis reduction, heat evaporation, electrochemical reduction (Siddiqi et al. 2018), chemical reduction (Karthik \& Geetha 2013), thermal decomposition (Joshi et al. 1998) etc have been extensively used for the synthesis of NPs in the nanotechnology. Out of various possible methods, chemical approaches are the most popular methods for the fabrication of nanoparticles. However, the chemical synthesis induces toxicity directly or indirectly to the environment. Hence, the developments of clean, biocompatible, non-toxic and eco-friendly methods for the synthesis of nanoparticles are extensively studied these days (Joseph et al. 2016; Aruoma et al. 2012).

Citrus medica Linn (commonly called as Lemon) is a species of small evergreen tree comprising a flowering plant of family Rutaceae, native to Asia. Lemon, a yellow fruit is well known for citric acid and ascorbic acid. It has been well known that lemon is of considerable significance from a medicinal point of view. Many of fatal diseases like cerebrovascular, cardiovascular, inflammation, cancer, problems of skin, throat, and asthma have been cured of by regular use of lemon water because of its antibacterial properties (Aruoma et al. 2012). Main objective of the present research is to carry out bio-synthesis copper nanoparticles (CuNPs) using locally available lemon extract to study effect of various parameters on synthesis of CuNPs and to evaluate its antibacterial activity against different strains.

\section{Materials and Method}

\section{Preparation of lemon extract}

The lemon extract was prepared just by squeezing the fresh, washed lemons into a clean container for the required amount and then filtered with muslin cloths first and then with Whatman No.1 filer paper.

\section{Bio-synthesis of copper nanoparticles (CuNPs)}

CuNPs were synthesized by simple and non-toxic, green methodology with slight modification using the protocol (Jayandran et al. 2015). $100 \mathrm{~mL}$ of $1 \mathrm{mM} \mathrm{CuSO}_{4}$ solution was allowed to react with $10 \mathrm{~mL}$ of lemon extract in a conical flask. Here, biomolecules present in lemon extract is used as reducing and self-stabilizing agents for the CuNPs. The reaction mixture was stirred at $60^{\circ} \mathrm{C}$ for 1 hour and the color changed from light blue to reddish dark brown color. Then after, the obtained precipitate was centrifuged at $4000 \mathrm{rpm}$ for $30 \mathrm{~min}$ and the supernatant liquid was decanted off. Finally, residue, CuNPs was washed with distilled water many times to remove impurities and collected after oven-dried at less than $80^{\circ} \mathrm{C}$. The process was repeated to study the effect of various parameters like temperature, concentration of precursor solution, and volume of the lemon extract in the synthesis process of CuNPs.

\section{Characterization techniques}

Preliminarily, the formation of nanoparticles was analyzed by visual observation of the changes in the color of the reacting solution. Then, the fabrication of CuNPs was confirmed using double beam UV-visible Spectrophotometer (LABTRONIC, Model LT-2802) in a range of wavelengths from 200 to $800 \mathrm{~nm}$ at interval of $5 \mathrm{~nm}$. Similarly, the Energy Dispersive X-ray (EDX) spectroscopic studies were carried out by using EDX-8000 SHIMADZU to explore the elemental compositions of the sample. FTIR analysis was carried by using FTIR - Tracer-100 to identify the functional groups of the biomolecules responsible for reduction of the precursor. The FTIR spectrum was recorded at the wave number range of $500-4000 \mathrm{~cm}^{-1}$ at resolution of $4 \mathrm{~cm}^{-1}$. Crystal morphology of the prepared sample was studied using XRD (Bruker D2 Phaser), at diffraction angle (20) range of $10^{\circ}$ to $80^{\circ}$ at the rate of $2 \% \mathrm{~min}$. Surface morphology of the sample was investigated using Field Emission Scanning Electron Microscopy (FE SEM, Carl Zeiss, SUPRA 40 VP, Oberkochen, Geramny). 


\section{Agar well diffusion method}

Antibacterial assay was performed by agar well diffusion method which measures mainly, zone of inhibition (ZOI) of the CuNPS against pathogenic bacteria. Firstly, a $2 / 3$ colony of bacteria was transferred and cultured into a Nutrient Broth (NB) and incubated at $37^{\circ} \mathrm{C}$ for 24 hours. After incubation, each stain was diluted with sterile distilled water. The turbidity of dilution was checked by comparing with 0.5 McFarland standards.

Then after, Muller Hilton Agar (MHA) plates were taken and labeled for each bacteria; Escherichia coli ATCC 25922, Staphylococcus aureus ATCC 25923 and Salmonella typhi. Then the sterile cotton swab was dipped into the test tube containing diluted bacterial stain and swabbed in-to the sterile Muller Hilton Agar (MHA) plates. The cotton swab was swabbed uniformly into the MHA plates. It was allowed to diffuse for about 20 minutes at the room temperatures. 5 wells of 4 mm were bored in the inoculated media with the help of sterile cork-borer $(4 \mathrm{~mm})$. The liquid sample was divided into three parts, which of them include $100 \%$ crude, $50 \%$ diluted solution, and $25 \%$ diluted solution. Each well was loaded with different extracts, positive control (Ofloxacin) $50 \mu \mathrm{L}$, negative control (DMSO) $50 \mu \mathrm{L}$, Crude liquid (100\%) $50 \mu \mathrm{L}$, liquid (50\%) $50 \mu \mathrm{L}$ and liquid (25\%) $50 \mu \mathrm{L}$. Then after, extract was set for diffusion for about 30 minutes at ordinary condition. Finally, the disc was incubated for 24 hours.

\section{Results and Discussion}

\section{Visual observation}

Upon visual observation, blue color was found to be changed to a shiny reddish-brown color indicating the reduction of $\mathrm{Cu}^{2+}$ to metallic copper of zerovalent $\left(\mathrm{Cu}^{0}\right)$. The preliminary result was found to be in good agreement with that of Kotval et al. (2018). It was further characterized by UV-visible spectroscopy for the confirmation of nanoparticles.

\section{UV-visible spectroscopy}

Result obtained from UV-vis spectroscopy of the bio-synthesized CuNPs and that of lemon extract is presented graphically in Figure 1. There is no any absorption peak in plot (a), UV-vis spectrum of neat lemon extract whereas the plot (b), the UV-vis spectrum of CuNPs (at volume ratio 1:10) shows a maximum absorption peak at around wavelength $565 \mathrm{~nm}$ which attributes the presence of CuNPs due to surface plasmon resonance of conducting electrons (Kotval et al. 2018; Chung et al. 2017).

Similarly, UV-visible spectra were used to study the effect of different parameters such as the volume of lemon extract, the concentration of $\mathrm{CuSO}_{4}$ solution, and temperature on bio-synthesis of CuNPs and the results are shown in next section.

\section{Effect of volume of the lemon extract on the bio-syn- thesis of CuNPs}

The UV-visible spectra of CuNPs synthesized using different volumes 10,20,30 and $50 \mathrm{~mL}$ of the lemon extract with fixed volume of precursor $(1 \mathrm{mM})$ are presented in Figure 2. Each of the plots shows presence of maximum absorption band at wavelength around $565 \mathrm{~nm}$, which is due to surface plasmon resonance of CuNPs. The peaks are shifted slightly towards higher wavelength from 565 to $590 \mathrm{~nm}$ and broadened with the increase in volume of the extract from 10 to $50 \mathrm{~mL}$. The higher the volume of extract, more reducing

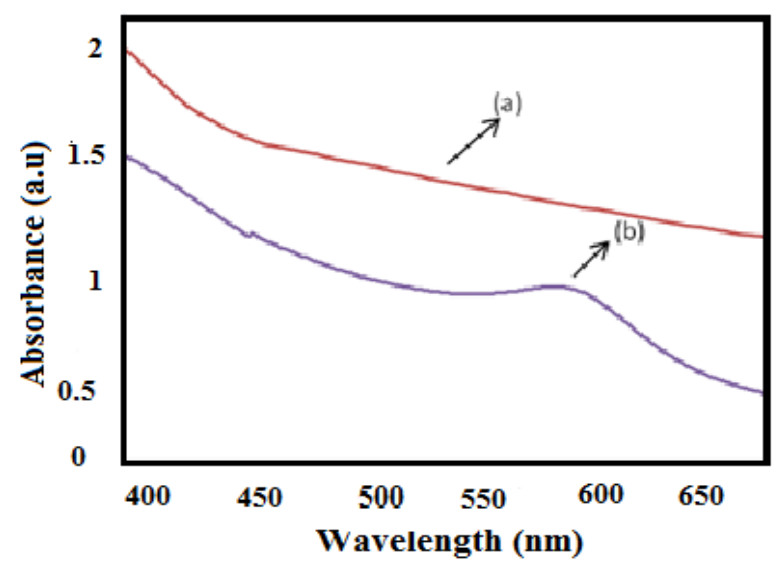

Fig 1: UV-visible spectra of (a) Lemon extract (b) as-synthesized CuNPs using lemon extract and $\mathrm{CuSO}_{4}(1 \mathrm{mM})$ of ratio $(1: 10)$ stirring at $60^{\circ} \mathrm{C}$.

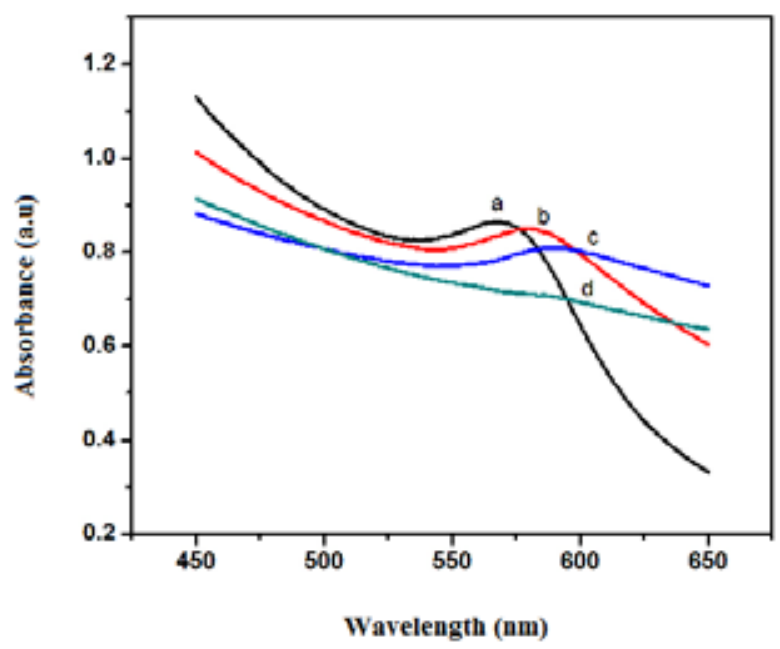

Fig 2: UV-visible spectra of Cu NPs prepared with $1 \mathrm{mM} \mathrm{CuSO}_{4}$ at different volume of lemon extract (a) $10 \mathrm{~mL}$ (b) $20 \mathrm{~mL}$ (c) $30^{4}$ $m L$ (d) $50 m L$. 
agent are available hence, more number of CuNPs are formed which soon forming agglomerated nanoparticles at higher volumes $(30 \& 50 \mathrm{~mL})$. The frequency and width of the absorption peak depend on the size and shape of the metal nanoparticles (Augustine et al. 2013).The absorption peak formed by low volume i.e $10 \mathrm{~mL}$ is intense due to formation of nanosized particles. Hence, this volume is found to be the optimum for the bio-synthesis.

\section{Effect of concentration of $\mathrm{CuSO}_{4}$ solution on the bio-synthesis of CuNPs}

Result of UV-visible spectra of CuNPs at concentration variation (Figure 3) shows that the absorption peak of minimum concentration i.e $1 \mathrm{mM}$ is more intense compared to that of higher concentration ( $5 \mathrm{mM}$ and $9 \mathrm{mM}$ ) because of the formation of smaller sized nanoparticles. With the increase in concentration of $\mathrm{CuSO}_{4}$, the peak position is slightly shifted towards higher wavelength and broadened also as more $\mathrm{Cu}^{2+}$ ions take part in reduction reaction and collision between nanoparticles accomplished leading to formation of agglomerated nanoparticles (Sekhar et al. 2016; Surmawar et al. 016). Thus, $1 \mathrm{mM}$ is referred as the optimum concentration for fabrication of CuNPs.

\section{Effect of temperature on the bio-synthesis of CuNPs}

It is a well known fact that temperature is being one of the significant parameters for carrying out any reaction. Bio-synthesis of CuNPs was carried out at different temperatures, i.e. at room temperature, $40^{\circ} \mathrm{C}, 60^{\circ} \mathrm{C}$, and $80^{\circ} \mathrm{C}$, respectively. Then, results of UVvisible spectroscopy of each sample are shown in Figure 4. The figure depicts that no absorption band appear at low temperatures i.e at room temperature and $40^{\circ} \mathrm{C}$ indicating low temperature is not favorable for the formation of nanoparticles. When the temperature is increased to $60^{\circ} \mathrm{C}$ and $80^{\circ} \mathrm{C}$, maximum absorption of the CuNPs are appeared at $570 \mathrm{~nm}$ and $585 \mathrm{~nm}$, respectively. The values are in the UV range of characteristic values of CuNPs as reported in literatures (Jayandran et al. 2015; Dinda et al.(2015). On comparing two UV peaks, curve $\mathrm{c}$ is comparatively sharper than the curve $\mathrm{d}$ indicating the presence of nanosized CuNPs. Hence, $60^{\circ} \mathrm{C}$ is found to be the favorable temperature for fabricating the CuNPs.

\section{Energy dispersive X-ray (EDX) spectroscopy}

Energy dispersive X-ray (EDX) spectroscopy is essential tool for achieving information about elemental composition. The Figure 5 shows the presence of energy absorption band at $8 \mathrm{keV}$ supporting the presence of copper as reported by Caroling et al. (2015). From the EDX analysis, the weight percentage of elemental copper is found to be $22.599 \%$. Hence, as-synthesized copper nanoparticles seemed associated with other elements remaining as impurities which may be obtained from the instrument and the sample preparation process.

\section{Fourier transform infrared spectroscopy (FTIR)}

The results of FTIR spectroscopy of bio-synthesized CuNPs using lemon extract is shown in Figure 6, which

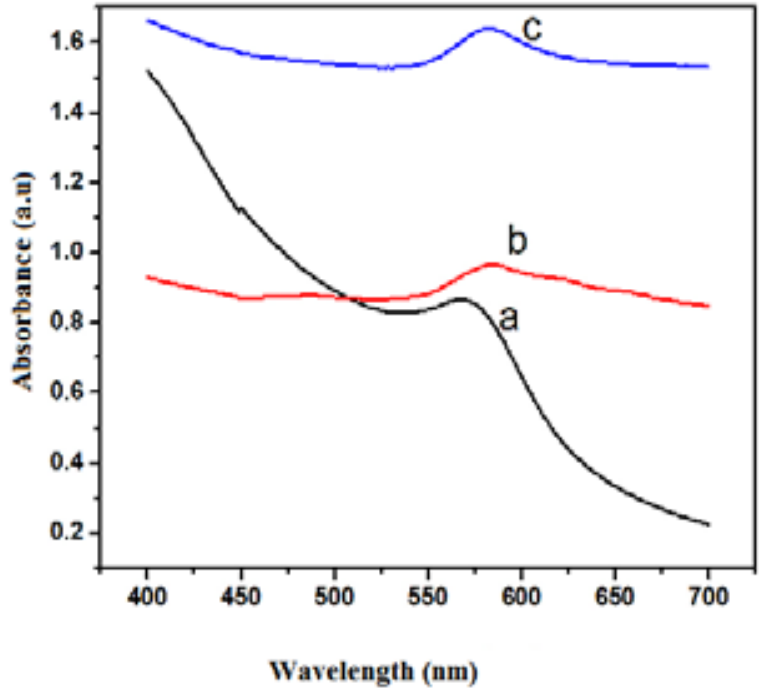

Fig 3: UV-visible spectra of CuNPs formed at different concentrations of $\mathrm{CuSO}_{4}$ solution (a) $1 \mathrm{mM}$ (b) $5 \mathrm{mM}$ (c) $9 \mathrm{mM}$

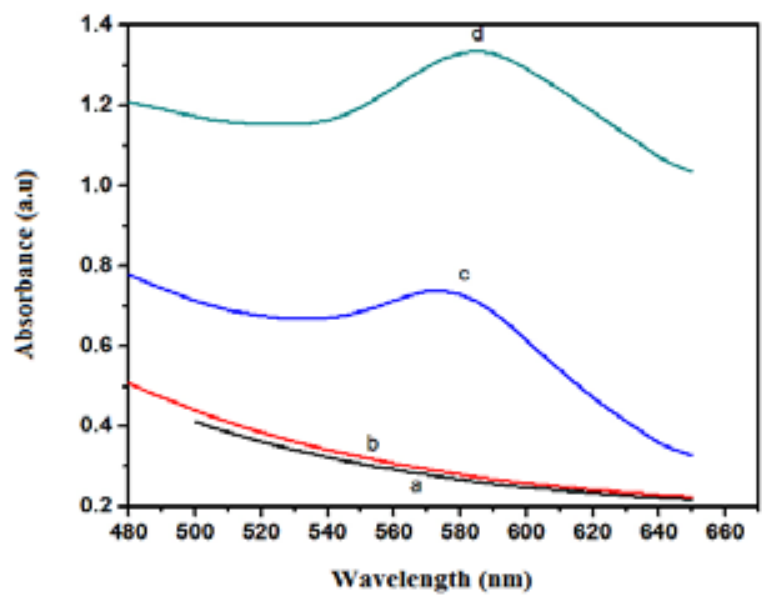

Fig 4: UV-visible spectra of biosynthesized CuNPs using lemon extract and $\mathrm{CuSO}_{4}$ at different temperature (a) room temperature, (b) $40^{\circ} \mathrm{C}$, (c) $60^{\circ} \mathrm{C}$ and (d) $80^{\circ} \mathrm{C}$

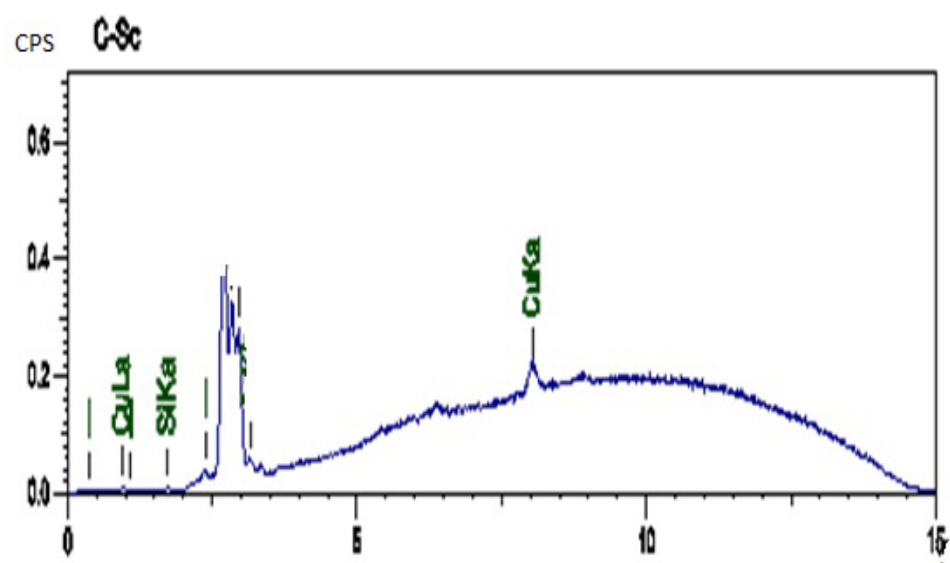

Fig 5: Energy Dispersive X-ray (EDX) Spectrum of as-synthesized CuNPs at $60^{\circ} \mathrm{C}$ 
illustrates the presence of various kinds of biomolecules surrounding the CuNPs. The absorption peak located at $3293 \mathrm{~cm}^{-1}$ corresponds to stretching vibrations of hydrogen bonded -OH groups. Further, peaks at $2900 \mathrm{~cm}^{-1}$ are due to symmetric \& asymmetric $\mathrm{C}-\mathrm{H}$ stretching of phenolic (Kumar et al. 2017), peaks observed at $2093 \mathrm{~cm}^{-1}, 1639 \mathrm{~cm}^{-1}, 1300 \mathrm{~cm}^{-}$ 1, $1450 \mathrm{~cm}^{-1}$ and $1022 \mathrm{~cm}^{-1}$ refers to $-\mathrm{CN}$ stretching, $-\mathrm{C}=\mathrm{C}$ relation, $-\mathrm{C}=\mathrm{O}$ stretching, $-\mathrm{OH}$ bending of phenolic group and $-\mathrm{C}-\mathrm{H}$ stretching characteristic of aromatic nucleus, respectively (Kumar et al. 2017; Trifunschi et al. 2015). While the undesirable peak observed $2300 \mathrm{~cm}^{-1}$ is due to instrumental error according to technician.

\section{X-Ray diffraction (XRD)}

$\mathrm{X}$-ray diffraction pattern of CuNPs is shown in Figure 7. The figure shows sharp peaks at diffraction angles $43.5^{\circ}$, $50.6^{\circ}$, and $74.0^{\circ}$ which are equivalent to the reflections revealed by different literatures. It depicts the presence of (111), (200) and (220) planes indicating formation of facecentered cubic (fcc) crystals of CuNPs which follows Joint Committee of Powder Diffraction Standards (File No.089-

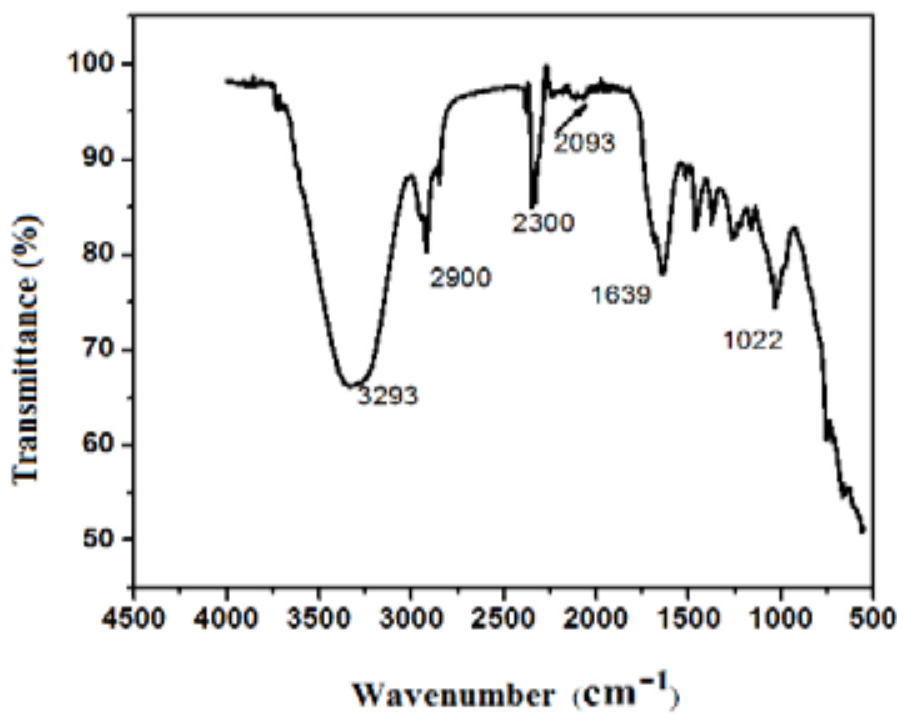

Fig6: FTIR Spectra of CuNPs synthesized from the lemon extract and $\mathrm{CuSO}_{4}$ solution (1:10)

2838) (Dinda et al. 2015; Suramwar et al. 2016; Samar et al. 2017; Kumar et al. 2017). Various other small peaks observed at $26^{\circ}, 31^{\circ}$ and $36^{\circ}$ may be due to formation of oxidized copper because sample was oven dried at less than $80^{\circ} \mathrm{C}$.

The average size of the crystallite is estimated by using the Debye-Scherrer equation (Carolling et al. 2015; Mittu 2016).

$$
\mathrm{D}=\frac{0.9 \lambda}{\beta \operatorname{Cos} \theta}
$$

Where ' $\lambda$ ' is wavelength of X-ray (0.1541 nm), ' $\beta$ ' is FWHM (full width at half maximum), ' $\theta$ ' is the diffraction angle, and ' $D$ ' is crystallite size. Substituting the corresponding values on the Scherrer equation, the average crystallite size was estimated to be $17.7 \mathrm{~nm}$.

\section{Field emission scanning electron microscopy (FE-SEM)}

Field Emission Scanning Electron Microscopy (FESEM) was employed to visualize the surface morphology of

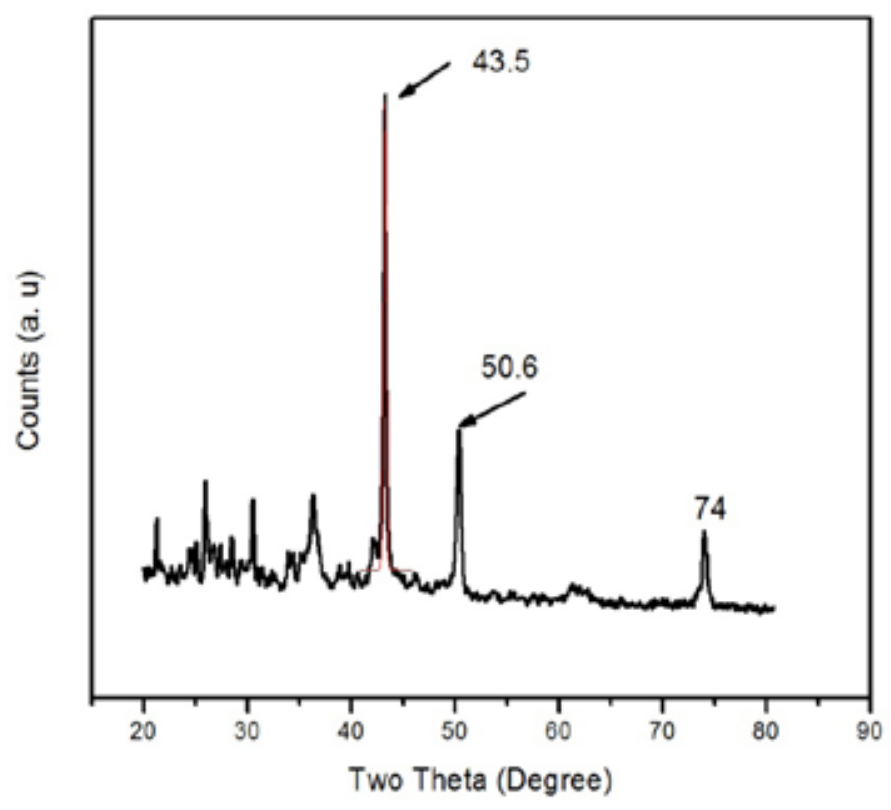

Fig7: X-ray diffraction pattern of bio-synthesized Copper nanoparticles at $2 \theta$ range from $10-80^{\circ}$. CuNPs. SEM images of CuNPs at different magnification are shown in Figure 8. SEM image Figure 8(a), lower magnification showed presence of white small grains of spherical and rod shape of CuNPs. Similarly, Figure 8(b), the higher magnification reveals clearly the presence of agglomerated nanoparticles distributed unevenly in a different direction. But it is difficult to calculate the size of these NPs. The size of NPs can be calculated using Transmission Electron Microscope (TEM). 

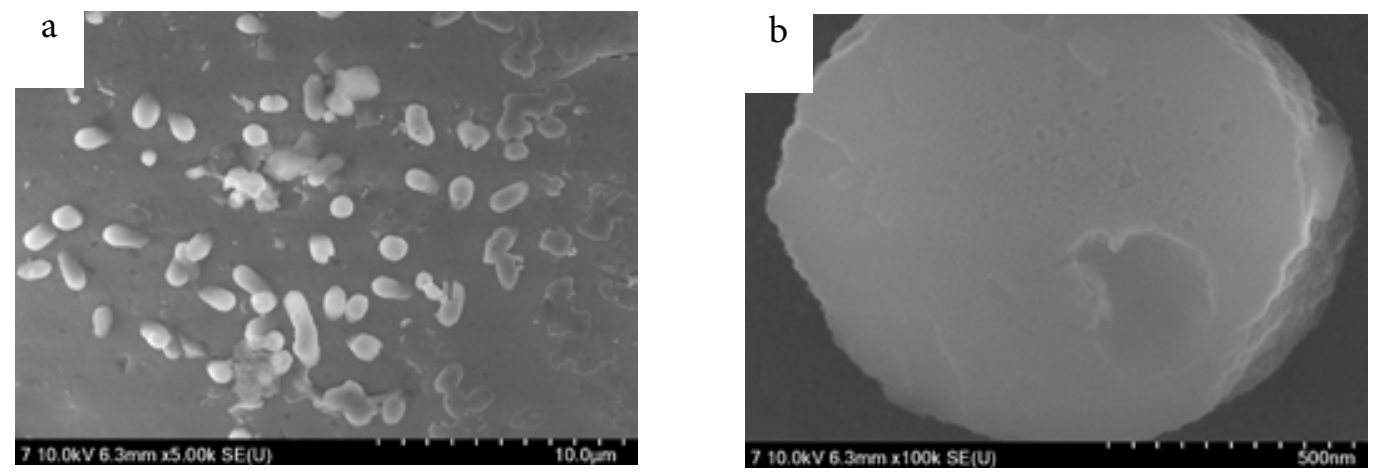

Fig 8: FE-SEM images of CuNPs at (a) lower magnification, (b) at higher magnification

\section{Antibacterial activity of copper nanoparticles}

The antibacterial activity of bio-synthesized CuNPs was evaluated against Staphylococcus aureus (Gram positive bacteria), Salmonella typhi and Escherichia coli (Gram negative bacteria), using agar well diffusion method. Commercial antibiotic, ofloxacin was used as positive control and Dimethyl sulfoxide (DMSO) was as negative control. Figure 9 presents snaps of zone of inhibition produced by $S$. aureus, $S$. typhi and E. coli at various concentrations, and result obtained is tabulated in Table 1 .
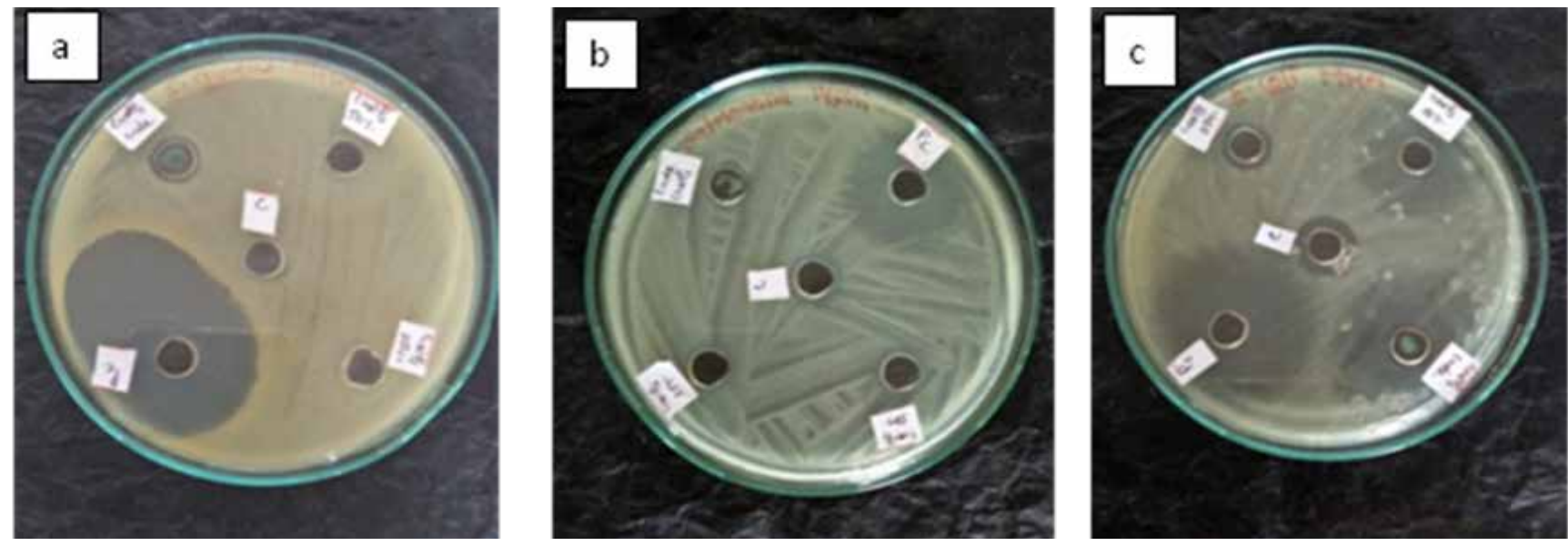

Fig 9: Zone of inhibition (ZOI) produced by agar well diffusion of bio-synthesized CuNPs against bacteria: (a) Staphylococcus. aureus (b) Salmonella typhi and (c) Escherichia coli

From the antibacterial test, ZOI of CuNPs of different concentrations is found greater for Gram negative bacteria than Gram positive bacteria due to differences in thickness of peptidoglycan layers (Theivasavthi \& Alagar 2011).

Table 1: Zone of inhibition (ZOI) of as-synthesized CuNPs and positive control ofloxacin

\begin{tabular}{c|c|c|c|c|c|c}
\hline \multirow{2}{*}{ S.N } & \multirow{2}{*}{ Test organisms } & \multicolumn{4}{|c}{ Zone of Inhibition } & \multirow{2}{*}{ Ofloxacin } \\
\cline { 3 - 6 } & & \multicolumn{4}{|c}{ CuNPs } \\
\cline { 3 - 6 } & Crude & $\mathbf{5 0 \%}$ & $\mathbf{2 5 \%}$ & $\begin{array}{c}\text { Control } \\
\text { (DMSO) }\end{array}$ & \\
\hline $\mathbf{1}$ & Staphylococcus aureus & $12 \mathrm{~mm}$ & $0 \mathrm{~mm}$ & $0 \mathrm{~mm}$ & $0 \mathrm{~mm}$ & $35 \mathrm{~mm}$ \\
\hline $\mathbf{2}$ & Salmonella typhi & $11 \mathrm{~mm}$ & $11 \mathrm{~mm}$ & $0 \mathrm{~mm}$ & $0 \mathrm{~mm}$ & $28 \mathrm{~mm}$ \\
\hline $\mathbf{3}$ & Escherichia coli & $\begin{array}{c}28.5 \\
\mathrm{~mm}\end{array}$ & $24 \mathrm{~mm}$ & $11 \mathrm{~mm}$ & $0 \mathrm{~mm}$ & $39 \mathrm{~mm}$ \\
\hline
\end{tabular}




\section{Conclusions}

Bio-synthesis of Copper nanoparticles (CuNPs) was carried out using lemon extract relatively at lower cost. The formation of CuNPs was confirmed by presence of characteristic UV-vis peak at $565 \mathrm{~nm}$. Bio-synthesis of CuNPs was found to be much affected by the various parameters such as volume of lemon extract, concentration of the precursor, temperature etc. From the experiment, optimum volume of lemon extract and concentration of precursor is found to be $10 \mathrm{~mL}$ and $1 \mathrm{mM}$, respectively. At the same time, the favorable temperature is referred to be $60^{\circ} \mathrm{C}$. Further, EDX spectroscopic analysis confirmed the presence of metallic copper with $22.59 \%$ by weight. Likewise, FTIR spectrum confirmed the presence of various biomolecules such as alkaloids, flavonoids, proteins at the surface of nanoparticles. Various reflections observed in XRD pattern revealed CuNPs are of purely crystalline nature and the average crystallite size is found to be of $17.7 \mathrm{~nm}$. Finally, agar well diffusion assay revealed distinct zone of inhibitions (ZOI) by CuNPs against different bacterial strains suggesting that CuNPs have potential antibacterial activity. Regarding future perspective, bio-synthesis using micro-organisms, study of effect of time, $\mathrm{pH}$ on synthesis, exploration of optical and catalytic properties of CuNPs are still of interesting topics for research in future. Additionally, the surface of CuNPs can also be decorated with other metal or metallic oxide nanoparticles for enhancement of properties of CuNPs.

\section{Acknowledgements}

Authors would like to thank University Grant Commission, Bhaktapur, Nepal for providing FRG 73-74 S\&T grant and Amrit Campus for providing space.

\section{References}

Aruoma, O. I., Landes, B., Ramful, B. D., Bourdon, E., Neergheen, B. V., Wagner, K.-H., and Bahorun, T. 2012. Functional Benefits of Citrus Fruits in the Management of Diabetes. Preventive Medicine. 54: 12-16.

Ashtaputrey, D. S., Ashtaputrey, D. P., and Yelane, N. 2017. Green Synthesis and Characterization of Copper Nanoparticles Derived from Murraya koenigii Leaves Extract. Journal of Chemical and Pharmaceutical Sciences.10(3): 1288-1291.

Augustine, R., Kalarikkal, N., and Thomas, S. 2013. Facile and Rapid Method for the Black Pepper Leaf Mediated Green Synthesis of Silver Nanoparticles and the Antimicrobial Study. Applied Nanoscience. DOI: 10.1007/s13204-013-0260-7.

Chung, I. M., Abdul Rahuman, A., Marimuthu, S., Kirthi, A. V., Anbarasan, K., Padmini, P. and Rajakumar, G. 2017. Green Synthesis of Copper Nanoparticles using Eclipta prostrata Leaves Extract and their Antioxidant and Cytotoxic Activities. Experimental and Therapeutic Medicine. 14(1): 18-24.

Caroling, G., Priyadharshini, M. N., Vinodhini, E., Ranjitham, A. M. and Shanthi, P. 2015. Biosynthesis of Copper Nanoparticles using Aqueous Guava Extract Characterisation and Study of Antibacterial Effects. International Journal of Pharmacy and Biological Sciences. 5(2): 25-43.

Dinda, G., Halder, D., Vázquez, C., López,Q, M., and Mitra, A. 2015. Green Synthesis of Copper Nanoparticles and their Antibacterial Property. Journal. Surface Science and Technology. 31: 117-122.

Ekezie, C. F. G., Suneetha, J. W., Maheswari, U. K., and Prasad, T. N. V. K. V., Kumari, A. B. 2017. Green Synthesis of Copper Nanoparticles using Momordica charantia fruit Extracts and Evaluation of their Anti-microbial Efficacy. International Journal of Current Microbiology and Applied Sciences. 6(4): 99-109.

Jayandran, M., Haneefa, M. M., and Balasubramanian, V. 2015. Green Synthesis of Copper Nanoparticles using Natural Reducer and Stabilizer and an Evaluation of Antimicrobial Activity. Journal of Chemical and Pharmaceutical Research. 7(2): 251-259.

Joseph, A. T., Prakash, P. and Narvi, S. S. 2016. Phytofabrication and Characterization of Copper Nanoparticles using Allium sativum and its Antibacterial Activity. International Journal of Science Engineering and Technology. 4(2): 463-472.

Karthik, D. A. and Geetha, K. 2013. Synthesis of Copper Precursor, Copper and its Oxide Nanoparticles by Green Chemical Reduction Method and its Antimicrobial Activity. Journal of Applied Pharmaceutical Science. 3(5): 16-21. 
Kotval, S. C., John, T. and Parmar, K. A. 2018. Green Synthesis of Copper Nanoparticles using Mitragyna parvifolia Plant Bark Extract and its Antimicrobial Study. Journal of Nanoscience and Technology. 4(4): 456-460.

Kulkarni, V. and Kulkarni, P. 2013. Green Synthesis of Copper Nanoparticles using Ocimum sanctum Leaf Extract. International Journal of Chemical Studies.1(3): 1-4.

Kumar, B., Smita, K., Cumba, L., Debut, A., Angulo, Y. 2017. Biofabrication of Copper Oxide Nanoparticles using Andean Blackberry (Rubus glaucus Benth.) Fruit and Leaf. Journal of Saudi Chemical Society. 21: 475-480.

Mittal, A. K., Chisti, Y. and Banerjee, U. C. 2013. Synthesis of Metallic Nanoparticles using plant Extracts. Biotechnology Advances. 31(2): 346-356.

Mittu, R. 2016. Synthesis, Characterization of Copper Nanoparticles - A Review. International Advanced Research Journal in Science, Engineering and Technology. 3(5): 37- 40.

Padma, N., Banu, S. and Kumari, S. 2018. Studies on Green Synthesis of Copper Nanoparticles using Punica granatum. Annual Research \& Review in Biology. 23: 1-10.

Prathna, C. T., Mathew, L., Chandrasekaran, N., Raichur, A. and Mukherjee, A. 2016. Biomimetic Synthesis of Nanoparticles: Science, Technology \& Applicability. Biomimetics Learning From Nature.1-23.

Siddiqi, K. S., Husen, A. and Rao, R. A. K. 2018. A Review on Biosynthesis of Silver Nanoparticles and their Biocidal Properties. Journal of Nanobiotechnology. 16(1): 1-14.

Samar, F., Kalainila, P., Ernest, R., Renganathan, S. 2017. Green Synthesis of Copper Nanoparticles from Passiflora foetida Leaf Extract and its Antibacterial Activity. Asian Journal of Pharmaceutical and Clinical Research. 10(4): 79-83.

Sekhar, C. E., Rao, K. K. S. V., Rao, M. K. and Kumar, P. S. 2016. A Green Approach to Synthesize Controllable Silver Nanostructures from Limonia acidissima for Inactivation of Pathogenic Bacteria. Cogent Chemistry. 2(1): 1-14.

Suramwar, V. N., Thakare, R. S. and Khaty, T. N. 2016. One Pot Synthesis of Copper Nanoparticles at Room Temperature and its Catalytic Activity. Arabian Journal of Chemistry. 9: 1807-1812.

Tu, H. L. 2019. Biosynthesis, Characterization and Photocatalytic Activity of Copper/Copper Oxide Nanoparticles Produced using Aqueous Extract of Lemongrass Leaf. Composite Materials. 3(1):30-35.

Thirugnanasambandan, T. and Alagar, M. 2010. X-Ray Diffraction Studies of Copper Nanopowder. Archives of Physics Research. 1(2): 112-117.

Thirugnanasambandan, T. and Alagar, M. 2011. Studies of Copper Nanoparticles Effects on Micro-organisms. Annals of Biological Research: 2(3): 368-373.

Trifunschi, S., Munteanu, F. M., Agotici, V., Ardeleanan, P. S., Gligor, R. 2015. Determination of Flavonoid and Polyphenol Compounds in Viscum album and Allium sativum Extracts. International Current Pharmaceutical Journal. 4(5): 382385. 